\title{
Ambulante Anästhesie: Grenzen und Möglichkeiten
}

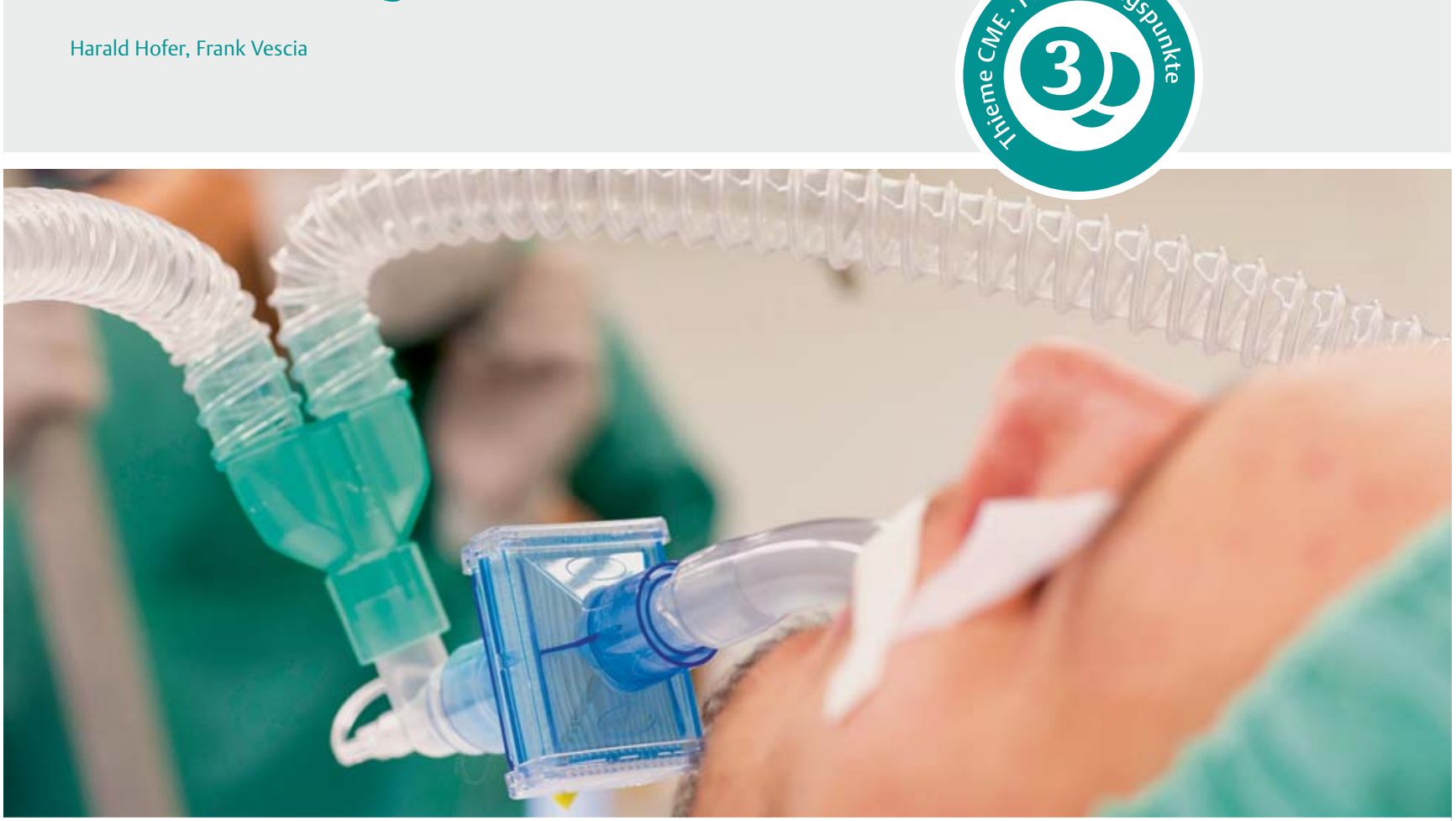

Quelle: KH Krauskopf.

Ambulante Operationen werden künftig zunehmend nachgefragt werden - Ursachen sind u. a. die alternde Bevölkerung und die finanzielle Lage der Krankenkassen. Der Beitrag geht ausführlich auf Begleiterkrankungen ein, bei denen die Entscheidung ambulant oder stationär sorgfältig abgewogen werden muss. Darüber hinaus nimmt er die betriebs- und volkswirtschaftlichen Grenzen und Möglichkeiten des ambulanten Operierens unter die Lupe.

\section{Einführung}

Seit der publikumswirksamen Einführung von Äther am 16. Oktober 1846 durch William Thomas Green Morton hat die Anästhesie eine erstaunliche Fortentwicklung durchschritten. Durch die Entwicklung moderner Anästhesietechniken, neuer Instrumente und Monitorverfahren, verbesserter Ausbildung, Patientenselektion und -evaluation wurden viele operative Prozeduren überhaupt erst möglich. Hinzu kommen eine verbesserte postoperative Überwachung und ein besseres postoperatives Schmerzmanagement. Ökonomische Gründe, Druck durch die Kostenträger und auch der Wunsch der Patienten, Krankenhausaufenthalte zu vermeiden, werden zu einer immer größeren Nachfrage nach ambulanten Ein- griffen führen. Schon 1918 schrieb Waters, ein Arzt, der sich auf dem Gebiet der ambulanten Anästhesie für Zahneingriffe in Sioux City, lowa, einen Namen gemacht hatte, bezüglich ambulanter Anästhesien: „The future of such a venture, I believe, is bright“ [1].

Hierfür ist es wichtig, die Möglichkeiten und Grenzen der ambulanten Anästhesie aufzuzeigen - dies jedoch ohne ein Dogma aufstellen zu wollen. Nicht jeder Anästhesist ist auf allen Feldern seines Tuns gleich ausgebildet und kompetent. Was für stationäre Einrichtungen gilt, gilt ebenso für das ambulante Anästhesieren: 


\section{Merke}

Die Grenzen des persönlichen Könnens sollten bei der ambulanten Anästhesie ebenso wenig überschritten werden wie die Grenzen, die uns der Patient vorgibt. Es gilt also für Anästhesisten und Chirurgen gleichermaßen: Know your limits!

\section{Umfelder und Szenarien}

Um über ambulante Anästhesie zu sprechen, lohnt es sich zunächst, das Umfeld zu beleuchten, in dem ambulante chirurgische Eingriffe durchgeführt werden:

- Ambulantes Operieren am Krankenhaus: Hier gibt es ein breites Spektrum von Settings, die von ambulanten Operationszentren am Krankenhaus bis hin zu „tagesstationären “ Eingriffen reichen. Dabei wird der ambulante Patient morgens aufgenommen, im Laufe des Tages operiert und geht dann abends nach Hause.

- Ambulante Operationszentren: Diese werden sehr häufig von Anästhesisten betrieben, die ihre Operationseinheiten an Operateure vermieten. Die Anästhesisten kümmern sich um die Vor- und Nachsorge der Patienten - soweit es nicht die eigentliche Operation betrifft.

- Ambulante Anästhesien in den Praxen niedergelassener Ärzte, die eigene Operations- oder Eingriffsräume vorhalten: Hier finden sich ganz unterschiedliche strukturelle Voraussetzungen - angefangen von Operationsräumen der Raumluftklasse $1 \mathrm{~b}$ mit dafür ausgelegten Aufwachräumen bis hin zu Eingriffsräumen, die für kleinere Eingriffe geeignet sind, größere Eingriffe aber nicht zulassen.

Details zu diesen Organisationsformen mag der Leser dem Artikel „Ambulante Anästhesie: Organisation in Praxis und Krankenhaus“ in dieser Ausgabe entnehmen.

Schon allein aus der Aufzählung dieser verschiedenen Settings wird deutlich, dass eine generelle Aussage zu den Grenzen und Möglichkeiten der ambulant durchgeführten Eingriffe nicht möglich ist. Trotzdem soll im Folgenden versucht werden, Aspekte zu beleuchten, welche die Grenzen des ambulanten Anästhesierens erkennen lassen.

\section{Ambulante Operationen}

Den in Deutschland umfassendsten Katalog ambulanter Operationen liefert der Einheitliche Bewertungsmaßstab (EBM), der die Vergütung für an der vertragsärztlichen Versorgung teilnehmende Ärzte regelt (s. „Gesetzliche Vorgaben“).

\section{GESETZLICHE VORGABEN}

\section{Grenzen ambulanter Operationen}

Schon in der Präambel zu Kapitel 31.2 des EBM sind Grenzen des ambulanten Operierens aufgezeigt: „Voraussetzung für die Berechnung der Gebührenordnungspositionen des Abschnittes 31.2 ist, dass die notwendigen sachlichen und personellen Bedingungen erfüllt sind...“ und „Der Operateur und der ggf. beteiligte Anästhesist sind verpflichtet, in jedem Einzelfall zu prüfen, ob Art und Schwere des beabsichtigten Eingriffs unter Berücksichtigung des Gesundheitszustandes des Patienten die ambulante Durchführung der Operation bzw. der Anästhesie nach den Regeln der ärztlichen Kunst mit den zur Verfügung stehenden Möglichkeiten erlauben und die erforderliche Aufklärung, Einverständniserklärung und Dokumentation erfolgt sind." [2].

Eng angelehnt an den EBM, jedoch nicht deckungsgleich, ist der Katalog nach § 115b SGB V. Hier wird eine Vielzahl ambulant durchführbarer Eingriffe aufgelistet, die im Regelfall auch im Krankenhaus zwingend ambulant durchgeführt werden sollen. Diese Verpflichtung führt häufig zum Streit zwischen Krankenhäusern und dem Medizinischen Dienst der Krankenkassen (MDK), wenn ein dort gelisteter Eingriff stationär durchgeführt wird. Des Weiteren werden auch im § 115b SGB V Qualitätskriterien genannt [3].

Verschiedene Fachgesellschaften geben ebenso Empfehlungen, welche Eingriffe ambulant durchführbar sind. Einen guten Überblick über diese Operationen gewinnt man auf der Homepage des Bundesverbandes Ambulantes Operieren e. V. (BAO) [4].

Diesen verschiedenen Katalogen ist gemein, dass sie nicht abschließend sind oder sein können. Zum einen führt der medizinische Fortschritt zu verändertem chirurgischem Vorgehen (endoskopische Eingriffe). Zum anderen bewirkt der ökonomische Druck, dass heute Eingriffe für ambulant durchführbar gehalten werden, die vor einigen Jahren noch zu einem mehrtägigen stationären Aufenthalt geführt hätten - z. B. Kreuzbandplastiken, Schultergelenkseingriffe etc.

Auch der aufgeklärte und mündige Patient fragt vielfach nach der Möglichkeit, einen Eingriff ambulant durchführen zu lassen. Vorteile für ihn sind [5]

- Verminderung nosokomialer Infektionsraten,

- geringe postoperative kognitive Dysfunktion,

- geringeres Thromboserisiko aufgrund der Frühmobilisation und

- bekanntes Setting, da die handelnden Personen ein Team bilden und dem Patienten oft bekannt sind. 


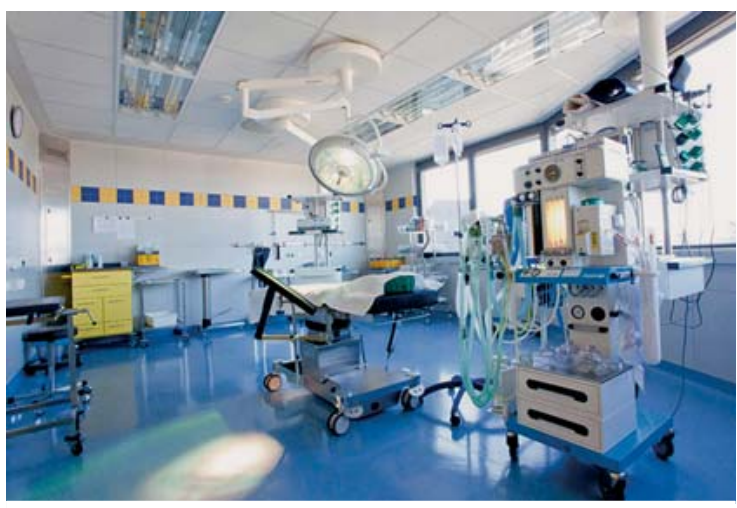

- Abb. 1 Operationssaal eines ambulanten Operationszentrums. Quelle: Tagesklinik am Brand, Mainz.

\section{Apparative Ausstattung}

Im Jahr 2012 wurden die Mindestanforderungen an den anästhesiologischen Arbeitsplatz durch den BDA und die DGAI verabschiedet und publiziert [6].

\section{Merke}

Bei den Mindestanforderungen an den anästhesiologischen Arbeitsplatz handelt es sich um Mindeststandards bezüglich ärztlichen Personals, Assistenzpersonals, apparativer Anforderungen, Räumen, Ausstattung und Einrichtungen.

Mindestanforderungen sind:

- Anästhesiesystem

- patientennahe Atemgasmessung

- Pulsoxymetrie

- EKG-Monitor

- Blutdruckmessung

Verfügbar sollten des Weiteren sein:

- Körpertemperaturmessung

- Defibrillator

- Relaxometrie, soweit Muskelrelaxanzien zum Einsatz kommen

- Blutzuckermessgerät

Empfohlen werden zudem ein Anästhesie-Beatmungsgerät und die oszillometrische Blutdruckmessung [6]. Darüber hinaus werden Besonderheiten in der Kinderanästhesie aufgelistet.

Einen Eindruck vom Operationssaal eines ambulanten Operationszentrums vermittelt $\boldsymbol{~ A b b . ~} \mathbf{1}$.

\section{Persönliche Kompetenz}

Blickt man in den Weiterbildungskatalog der zuständigen Landesärztekammern, so soll der angehende Facharzt für Anästhesie eine Vielzahl von Fertigkeiten erwerben und dann auch möglichst gut beherrschen. Dies ist aus der Sicht der Autoren ein verbreiteter Irrtum. Mit der bestandenen Facharztprüfung dokumentiert der Anästhesist lediglich ein gutes Verständnis für anästhesiologische Belange verbunden mit handwerklichen Fähigkeiten. Diese erlauben ihm, sich auf bestimmten Gebieten der Anästhesie eigenständig zu entwickeln und seine Kenntnisse zu vertiefen. Es wäre somit eine Selbstüberschätzung, wenn man als Facharzt jederzeit ambulante Anästhesien bei allen nachfolgend beschriebenen Patientengruppen anbietet und durchführt. Gerade das Wort „ambulant“ verleitet oft dazu, die anästhesiologische Tätigkeit als „leicht“ oder „mache ich eben mal“ zu klassifizieren - ein weiterer Irrtum, wie dieser Artikel zeigen soll. Der bei allen Operationen einzuhaltende Facharztstandard führt nicht automatisch zur Beherrschung aller Narkoseformen. Ebenso wenig beinhaltet er die Kenntnis aller Facetten des ambulanten Operierens, wie in der „Vereinbarung zur Qualitätssicherung ambulante Anästhesie“ [7] der DGAI beschrieben.

\section{Der Patient}

\section{Durchführbarkeit der ambulanten Anästhesie}

Im Mittelpunkt der Überlegungen zur Durchführbarkeit der ambulanten Anästhesie steht die Untersuchung des Patienten einschließlich Evaluation der Befunde im Kontext mit dem zu erwartenden Narkoseverfahren.

\section{FALLBEISPIEL}

\section{Entscheidungsfindung}

Es stellt sich eine durchaus aufgeklärte Patientin vor, die einen ambulanten Eingriff wünscht, z. B.

- eine Abrasio oder

- eine Leistenhernien-Operation oder

- eine kosmetische Bauchdeckenstraffung.

Der Operateur hat schon zugestimmt. Bei der Untersuchung zeigt sich eine adipöse Patientin (BMI = 38) nach Versorgung mit einem Koronarstent vor 2 Jahren, mit einer eingeschränkten Belastbarkeit, jedoch immer noch in der Lage, 3 Stockwerke zu Fuß zurückzulegen. Es bestehen eine prädiabetische Stoffwechsellage und eine chronische Bronchitis bei Nikotinabusus.

Oft muss der Anästhesist an dieser Stelle seine Entscheidung - ambulant oder stationär - mehr auf seine Erfahrung und die Kenntnis des Operateurs als auf objektive Kriterien stützen. Mögliche Entscheidungen finden sich am Ende dieses Teilkapitels (s. Fallbeispiele „Ambulante OP möglich“ und „Ambulante OP fraglich“).

Obwohl sie schon über 10 Jahre alt sind, lohnt sich ein Blick auf die Kriterien, die ein US-amerikanischer Berufshaftpflichtversicherer für ambulante Operationen aufgestellt hat. Gerade von einer Versicherungsgesellschaft 
sind risikoaverse Guidelines zu erwarten, sodass die folgende Einteilung eher überrascht:

- Patienten über 15 Jahre (ASA I-ASA III) sind als Kandidaten für ambulante Eingriffe geeignet.

- Neonaten (0-30 Tage), Säuglinge unter 6 Monaten und ASA-III-Kinder jeden Alters sollten Narkosen nur in entsprechend ausgestatteten Settings erhalten d.h. mit der Möglichkeit, alle potenziell auftretenden Komplikationen sofort zu behandeln und im Idealfall zu beherrschen.

- Kinder über 6 Monate bis 14 Jahre mit ASA-I- oder -II-Status können ambulant operiert werden, soweit alle räumlichen, apparativen und personellen Voraussetzungen stimmen. Dies trifft jedoch nicht zu für ehemalige Frühgeborene vor der 37. SSW im 1. Lebensjahr, da postoperativ die Gefahr von Apnoe immer gegeben ist [8].

\section{Merke}

Die Einteilung mag für den normalgewichtigen gesunden Patienten vernünftig sein, erscheint aber im Lichte einer immer älteren und damit mit Komorbiditäten behafteten Bevölkerung vereinfacht.

Auch das Mindestalter ist immer wieder ein Diskussionspunkt, wenn es um ambulante Eingriffe geht. Aufgrund der Adaptation von Stoffwechselabläufen nach der Geburt wird in Deutschland empfohlen, nur Kinder, die älter als 6 Monate sind, ambulant zu operieren. Ob die Grenze von 6 Monaten zu niedrig angesetzt ist, muss der ausführende Anästhesist selbst beurteilen. Unabdingbar für diese Kindernarkosen sind natürlich das Vorhalten von altersgerechtem Equipment (Defibrillator, Infusionslösungen, Material für intraossären Zugang - jeweils kindgerecht) und einer Hilfskraft, die in Kindernarkosen erfahren ist [6].

\section{FALLBEISPIEL}

\section{Monate altes Kind mit LKG}

Ein 6 Monate altes Kind mit einer Lippen-Kiefer-Gaumenspalte (LKG) soll 2-zeitig operiert werden: zunächst mit 6 Monaten der Verschluss der Lippe, mit 18 Monaten der Verschluss der Gaumenspalte. Kinder mit diesem Krankheitsbild haben gehäuft Herzvitien. Daran muss gedacht werden und dies muss zwingend durch einen Kinderkardiologen abgeklärt werden. Wenn sich hier keine pathologischen Befunde ergeben und das Kind auch sonst gesund ist, kann aus Sicht des Autors zumindest der Verschluss der Lippenspalte ambulant erfolgen. Zur Sicherung der Atemwege ist in diesem Fall nicht einmal eine Intubation nötig, eine Laryxmaske erscheint vollkommen ausreichend. Wichtigste Voraussetzungen hierfür sind - neben der entsprechenden kindgerechten Infrastruktur - ein erfahrener Anästhesist, ein erfahrener Operateur und das geübte Zusammenspiel dieser beiden Ärzte.

\section{Häufige Befunde und Erkrankungen}

Ein guter Überblick über mögliche Vorerkrankungen und die entsprechenden Bewertungen findet sich in der Empfehlung der DGAI „Präoperative Evaluation erwachsener Patienten vor elektiven, nicht kardiochirurgischen Eingriffen“ [9]. Häufige Befunde und Erkrankungen werden nachfolgend dargestellt.

\section{Respiratorische Erkrankungen wie}

Asthma bronchiale oder COPD

Ein Patient mit gut eingestelltem Asthma bronchiale oder einer nicht floriden chronisch obstruktiven Lungenerkrankung (COPD) kann durchaus für eine ambulante Operation in Betracht kommen. Die Entscheidung dazu muss in Abhängigkeit vom körperlichen Untersuchungsbefund, der bevorstehenden Operation (Dauer, Lagerung, postoperativ schmerzbedingte Atemhemmung) getroffen werden. Sehr häufig sind bei diesen Patienten Komorbiditäten wie Herzinsuffizienz, Hypertonie, koronare Herzerkrankung (KHK) vorhanden sowie ein Nikotinabusus. Bei höherem Grad einer COPD muss die Entscheidung für eine ambulante Operation wohl durchdacht werden und ist insbesondere von der Länge der geplanten Operation abhängig.

\section{Merke}

Eine Verbesserung der COPD-Symptomatik kann und sollte präoperativ in Zusammenarbeit mit dem Hausarzt oder Pulmologen erreicht werden [10].

\section{Kardiovaskuläre Vorerkrankungen}

Patienten mit deutlich eingeschränkter Herzleistung, ggf. verbunden mit einer Aortenstenose, sind sicherlich keine Kandidaten für einen ambulanten Eingriff.

Eine KHK, die beim Patienten keine weiteren Symptome hervorruft, ist ebenso wenig ein Ausschlusskriterium wie eine Koronarstent-Versorgung. Es müssen jedoch die Zeiten zwischen Implantation und Operation gemäß den Leitlinien eingehalten werden: Beim Bare Metal Stent (BMS) mindestens 30-45 Tage, beim Drug eluting Stent (DES) sollte mindestens 1 Jahr bis zur elektiven Operation vergangen sein [9]. Manche Studien belegen sogar für den BMS, dass ein erhöhtes Risiko bei elektiven Operationen für das gesamte 1. Jahr nach Stent-Implantation besteht [11].

Die Eignung eines Patienten mit einer kardialen Vorerkrankung für eine ambulante Operation sollte nicht nur von objektiven Befunden abhängig gemacht werden - auch die allgemeine Belastbarkeit muss berücksichtigt werden. Sicherlich ist dies manchmal eine subjektive Entscheidung des Anästhesisten, aber anhand der folgenden Kriterien durchaus objektivierbar. Die Belastbarkeit dieser Patienten lässt sich gut anhand des metabolischen Äquivalents (MET) ( Tab. 1) beschreiben. Ein MET-Wert größer oder gleich 4 wird als ausreichend für kleinere ambulante Eingriffe angesehen [12]. 
> Tab. 1 Funktionelle Leistungskapazität.

\begin{tabular}{|l|l|}
\hline MET & Aktivität \\
\hline 1 & kann selbstständig essen, trinken, Toilette benutzen \\
\hline 3 & kann 1-2 Blöcke in langsamer Geschwindigkeit laufen \\
\hline 4 & $\begin{array}{l}\text { kann leichte Hausarbeit erledigen und 2 Etagen Treppen } \\
\text { steigen, schwere Möbel verschieben }\end{array}$ \\
\hline 10 & $\begin{array}{l}\text { kann mäßig belastende Sportarten (Bowling, Golf) betreiben } \\
\text { kann anstrengende Sportarten (Fußball, Leichtathletik, } \\
\text { Skifahren) betreiben }\end{array}$ \\
\hline MET: metabolisches Äquivalent \\
\hline
\end{tabular}

- Tab. 2 Gewichtsklassifikation von Erwachsenen anhand des BodyMass-Index (BMI).

\begin{tabular}{|l|l|}
\hline BMI & Bewertung \\
\hline$<18,5$ & Untergewicht \\
\hline $18,5-24,9$ & Normalgewicht \\
\hline $25,0-29,9$ & Übergewicht im Stadium der Präadipositas \\
\hline $30,0-34,9$ & Übergewicht im Stadium der Adipositas Grad I \\
\hline $35,0-39,9$ & Übergewicht im Stadium der Adipositas Grad II \\
\hline$\geq 40$ & extreme Adipositas (Adipositas Grad III, Adipositas permagna) \\
\hline
\end{tabular}

Eine Hypertonie - oft schon behandelt - stellt man bei vielen Patienten jenseits des 60. Lebensjahres fest; sie ist per se sicher kein Ausschlusskriterium für eine ambulante Operation. Dem Blutdruck muss bei dieser Patientengruppe jedoch vermehrt Aufmerksamkeit gewidmet werden, damit ein zu starkes Absinken verhindert wird. In Kombination mit einer Karotisstenose kann eine solche ungewollte Hypotonie schwerwiegende Komplikationen (Apoplex, Erblindung etc.) auslösen, sodass ggf. ein stationärer Aufenthalt empfohlen werden sollte.

Merke

Die Komplikationshäufigkeit bei Risikopatienten ist stationär vermutlich nicht geringer als ambulant, die Möglichkeiten der Therapie unter intensivmedizinischen Bedingungen sind jedoch deutlich besser.

\section{Adipositas}

Adipositas ist eine Volkskrankheit, die in den letzten Jahren nicht nur bei Erwachsenen, sondern auch bei Kindern zunehmend beobachtet wird. Üblicherweise wird der Body-Mass-Index (BMI) zur Beschreibung herangezogen ( $\triangleright$ Tab. 2). Hierbei wird das Körpergewicht durch die Körperlänge zum Quadrat dividiert [13].

Um es vorwegzunehmen: Es muss dem Leser und dem jeweils ambulant tätigen Anästhesisten überlassen bleiben, ab welchem Grad der Adipositas er einen Eingriff als nicht ambulant durchführbar ansieht. In der Literatur finden sich zwar Expertenmeinungen zu diesem Thema, jedoch wenig harte Fakten [14]. Ein erhöhter BMI ist per se keine Kontraindikation für einen ambulanten Eingriff. Im Zusammenhang mit einer Adipositas permagna muss jedoch an Folgendes gedacht werden:

- Die Lungenfunktion ist reduziert und wird durch die Narkose noch weiter verschlechtert.

- Es findet sich gehäuft eine verschlechterte Funktion von Herz, Leber, Nieren, Stoffwechsel und Gerinnung. Erhöhtes Diabetes- und Thromboserisiko!

- Je nach Medikament muss die Dosierung dem Idealoder Normalgewicht angepasst werden.

- Es gibt häufig Intubationsschwierigkeiten aufgrund der Adipositas.

- Manche Operationstische sind nur bis 125 kg zugelassen, manch andere bis $450 \mathrm{~kg}$.

- Nicht nur die Operationstische müssen für dieses Gewicht zugelassen sein, sondern auch die Liegen/Betten in den Aufwachräumen.

Recherchiert man die einschlägigen Publikationen, so werden in ambulanten Operationseinrichtungen BMIWerte von 40 bzw. 50 als Grenze für eine ambulante Durchführung gezogen. Interessanterweise neigen deutsche Anästhesisten an dieser Stelle zu einem deutlich restriktiveren Vorgehen als US-amerikanische Kollegen [15].

\section{Adipositas und OSAS}

Sehr häufig vergesellschaftet mit der Adipositas ist das obstruktive Schlafapnoe-Syndrom (OSAS), insbesondere bei Männern ab 40 Jahren. Obwohl harte Daten fehlen, gibt es Expertenmeinungen, dass postoperative Risiken und Komplikationen bei diesen Patienten erhöht sind [16].

Im Gegensatz zu den Leitlinien anderer Organisationen befasst sich die Leitlinie der American Society of Anesthesiology (ASA) auch mit dem ambulanten Sektor. Allerdings ist die Empfehlung für ambulante OSAS-Patienten recht ungenau und knapp: So heißt es, vor dem operativen Eingriff solle festgelegt werden, ob die Prozedur besser ambulant oder stationär erfolgen sollte - dies anhand verschiedener Kriterien, die den Patienten, den Eingriff, die Anästhesie und die Möglichkeiten des ambulanten Bereichs berücksichtigen [17]. Die wichtigsten Kriterien [18] hierbei sind

- Grad der Schlafapnoe,

- anatomische Besonderheiten,

- die Schwere von Komorbiditäten,

- welcher Eingriff vorgenommen werden soll,

- die Art der Narkose und der Bedarf von Opioiden in der postoperativen Phase sowie

- ob der Patient postoperativ lückenlos überwacht werden kann.

Als postoperative Überwachungsmaßnahmen sollte bei adipösen Patienten [19]

- die Sauerstoffsättigung besonders engmaschig kontrolliert werden, 
- eine CPAP-Beatmung (Continuous positive Airway Pressure) schon im Aufwachraum möglich sein,

- Kopf und Oberkörper erhöht gelagert werden,

- der Gebrauch von Sedativa oder zentral wirksamen Analgetika postoperativ auf das Notwendigste beschränkt werden,

- auf die Anzeichen einer fortschreitenden Herzinsuffizienz, eines Thrombosegeschehens und eingeschränkter Atmung geachtet werden.

Merke

OSAS-Patienten mit schlecht eingestellten Begleiterkrankungen sollten nicht ambulant operiert werden. Sind OSAS und Begleiterkrankungen gut eingestellt und kann der Patient postoperativ am CPAP-Gerät atmen, spricht nichts gegen eine ambulante OP.

Entscheidend für den postoperativen Outcome ist u.a. die Compliance des Patienten (CPAP, Medikamenteneinnahme).

\section{FALLBEISPIEL}

\section{Adipositas permagna}

Ein stark adipöser Patient mit BMI größer 45 soll an einer Leistenhernie operiert werden. In der Anamnese gibt er eine diagnostizierte Schlafapnoe an und einen insulinpflichtigen Diabetes mellitus. Für die Schlafapnoe ist der Patient mit einer CPAP-Maske versorgt, die er jede Nacht trägt. Der insulinpflichtige Diabetes ist gut eingestellt, der Patient ist gut über seine Erkrankungen aufgeklärt. Hier spricht aus der Sicht des Autors nichts gegen eine ambulante Operation. Die Operation wird offen unter Verwendung von alloplastischem Material durchgeführt. Um postoperativ zentral wirkende Analgetika möglichst zu vermeiden, wird die Wunde mit Lokalanästhetika durch den Operateur umspritzt. Postoperativ werden nur peripher wirkende Analgetika gegeben, eine CPAP-Maske wird im Aufwachraum für 30 Minuten verwendet. Aufgrund des Einsatzes von Propofol und Remifentanil als Anästhetika ist der Patient schnell wach und atmet suffizient bei Oberkörperhochlagerung. Der Blutzucker wird vor Entlassung kontrolliert.

\section{Stoffwechselerkrankungen}

Hier ist natürlich an erster Stelle der Diabetes - entweder nicht insulinabhängig (NIDDM) oder insulinabhängig (IDDM) - zu nennen: eine Volkskrankheit, vergesellschaftet mit der Adipositas. Ein gut eingestellter Diabetes rechtfertigt auf keinen Fall eine Entscheidung gegen eine ambulante Durchführung der Operation. Es gibt verschiedene Regime, den IDDM-Patienten am Operationstag mit Insulin zu versorgen. Wichtig ist in erster Linie die Möglichkeit, den Blutzuckergehalt intra- und post- operativ zu bestimmen, um Hypo- oder schwerwiegende Hyperglykämien auszuschließen [9].

Schilddrüsenerkrankungen spielen für elektive Eingriffe eine eher untergeordnete Rolle.

\section{Weitere Ausschlusskriterien für ambulante Operationen}

Sicher gibt es noch weitere Ausschlusskriterien zur Durchführung ambulanter Anästhesien, die

- entweder selbsterklärend sind oder in der Praxis eine eher untergeordnete Rolle spielen (z. B. seltene Syndrome mit Missbildungen insbesondere im Bereich der Atemwege, seltene Muskelerkrankungen) bzw.

- eher weiche Faktoren in der Entscheidung gegen eine ambulante Narkose darstellen.

In der Literatur werden an verschiedenen Stellen genannt [20,21]:

- Gerinnungsstörungen

- akute Infektionen

- zerebrale Eingriffe

- Thoraxeingriffe

- erwarteter Blutverlust $>500 \mathrm{ml}$

- schlecht eingestelltes Epilepsieleiden

- Drogenkonsum

- lange Operationsdauer

\section{FALLBEISPIEL}

\section{Kind mit Infektionsverdacht}

Bei einem 2-jährigen Kind soll ein zahnärztlicher Eingriff in Vollnarkose durchgeführt werden. Geplant sind Füllungen und einige Zahnextraktionen. Es präsentiert sich ein weinerliches Kind mit gerötetem Kopf, jedoch ohne Fieber. Die Eltern geben an, dass das Kind seit einigen Tagen verschnupft sei und die Nase läuft. Zudem hätten sich die Eltern an diesem Tag extra freigenommen, sodass sie beide auf das Kind aufpassen könnten. An dieser Stelle entwickelt sich ein gewisser Druck auf den Anästhesisten. Die Zahnsanierung ist sicher ein elektiver Eingriff und kann auch zu einem späteren Zeitpunkt durchgeführt werden. Das Kind macht einen kränkelnden Eindruck, auch wenn keine schwerwiegenden Infektzeichen vorhanden sind. Die Eltern haben sich auf den ambulanten Eingriff zeitlich eingestellt. Für einen zahnärztlichen Eingriff ist eine Intubationsnarkose das Mittel der Wahl. Bei dem Kind kann allerdings ein Infekt der oberen Luftwege nicht ausgeschlossen werden und dies kann postoperativ nach Extubation zu Atemwegsproblemen führen. Daher sollte der Eingriff trotz der Erwartungshaltung der Eltern verschoben werden. Hierzu bedarf es aber einer gewissen Erfahrung, um diese patientenindividuelle Entscheidung zu treffen, und eines „Standings“ gegenüber den Eltern. Eine ambulante Narkose sollte zum jetzigen Zeitpunkt nicht durchgeführt werden.

Der $§ 115 b$ nennt weitere Kriterien, die für einen stationären Eingriff sprechen, wobei es sich auch hier eher um 
Anhaltspunkte handelt. Fehlende Kommunikationsmöglichkeiten mit dem Patienten oder fehlende sachgerechte Versorgung im Haushalt des Patienten sind an erster Stelle genannt. Die Schwere der Erkrankung (z. B. Bewusstlosigkeit, Verwirrtheit, akute Lähmung, akute Blutung) oder ein erhöhter Behandlungsaufwand (z. B. kontinuierliche intensive Überwachung, bedrohliche Infektionen) sind weitere dort aufgeführte Kriterien [3].

Gerade der Punkt „lange Operationsdauer“ führt immer wieder zu Diskussionen. Was ist „lang“? Während es seitens der Fachgesellschaften in Deutschland keine Richtlinien gibt, hilft wiederum ein Blick in die USA. Pennsylvania und Florida haben die Operationszeit in ambulanten Einrichtungen per Verordnung auf 4 Stunden limitiert [22]. Viele Surgical Center limitieren die OP-Zeit auf freiwilliger Basis auf 4, 5 oder auch 6 Stunden.

\section{Merke}

Insgesamt sind Komorbiditäten, der zu erwartende Blutverlust, Flüssigkeitsverschiebungen, die Operation per se und die postoperative Schmerztherapie ebenso wesentliche Faktoren wie die Operationsdauer.

Je länger die Operationsdauer, desto mehr muss aber geachtet werden auf:

- Blasenkatheter: ohne wohl schwer machbar, verbunden mit den üblichen Risiken: Via falsa, Infektionen, Blutungen

- Wärmemanagement: Wärmematten, vorgewärmte Infusionen

- Lagerung zur Vermeidung von Lagerungsschäden, Druckläsionen etc.

\section{Postoperative Phase}

In der unmittelbaren postoperativen Phase sollte es im ambulanten Setting keinen Unterschied in der Überwachung im Vergleich zum Krankenhaus geben. Dann wird der Patient nach einer gewissen Überwachungszeit nach Hause entlassen.

Die postoperative Überwachungszeit ist aus gutem Grund weder von der Fachgesellschaft noch durch Kapitel 31 des EBM festgelegt. Lediglich eine Ziffer im EBM erfordert eine Mindestüberwachungszeit. In der GOÄ finden sich Mindestzeiten, die sich aber nur auf die Abrechenbarkeit einzelner Gebührenordnungspositionen beziehen und nicht medizinisch fundiert sind.

Den Entlassungszeitpunkt in das häusliche Umfeld bestimmt der Anästhesist in Kooperation mit dem Operateur - denn auch dieser ist für sein Tun verantwortlich und muss potenzielle Gefahren vom Patienten abwenden (Nachblutungen, Verband etc.). Hierbei sind die entsprechenden Vereinbarungen und Empfehlungen der jeweiligen Fachgesellschaften zu berücksichtigen [7,23].
Der Patient muss im Vorfeld des Eingriffs schon darüber aufgeklärt worden sein, dass eine häusliche Betreuung zumindest bis zum nächsten Tag unbedingt gewährleistet sein muss. In den meisten Fällen ist dies durch den Lebenspartner oder die Kinder auch sichergestellt. Zudem muss gewährleistet sein, dass der Patient nicht mehr aktiv am Straßenverkehr teilnimmt. In Deutschland ist es üblich, hier einen Zeitraum von 24 Stunden zu fordern. Obwohl harte Daten für diese Regelung nicht vorliegen, gibt die Rechtsprechung für diesen Zeitraum dem Anästhesisten aber eine Mitschuld am Handeln des Patienten. Interessanterweise sind die den Autoren bekannten Gerichtsentscheide alle mit dem Medikament Midazolam verbunden. Bei Verwendung dieses Medikaments zur Prämedikation oder intraoperativ ist somit erhöhte Vorsicht, ggf. eine längere postoperative Überwachung, notwendig [24].

Es sei dahingestellt, ob der Patient in der Obhut einer verständigen Person besser oder schlechter überwacht wird als auf einer Station im Krankenhaus - eine Nachtschwester muss dort eine Vielzahl von Patienten versorgen.

\section{FALLBEISPIEL}

\section{Ambulante OP möglich}

An dieser Stelle soll nun die Frage beantwortet werden, die eingangs hinsichtlich der oben beschriebenen Patientin (s. „Fallbeispiel - Entscheidungsfindung“) gestellt wurde: Kann sie bei den bestehenden Komorbiditäten ambulant operiert werden?

Abrasio: ein Eingriff, der üblicherweise in 5-10 Minuten durchgeführt wird. In diesem Fall wird ein erfahrener ambulant tätiger Anästhesist trotz der Adipositas $(B M I=38)$ vermutlich keinen Grund sehen, diese Operation nicht ambulant durchzuführen. Die Begleiterkrankungen wie chronische Bronchitis, die prädiabetische Stoffwechsellage und auch die vor 2 Jahren erfolgte Stent-Implantation stellen keine Kontraindikationen für die ambulante Durchführung dar. Solange der gynäkologische Operationstisch für das Gewicht zugelassen ist, sollte der Eingriff aus Sicht des Autors auf jeden Fall ambulant erfolgen.

Verschluss einer Leistenhernie: Hier dürfte die Operationsdauer üblicherweise zwischen 20 und $45 \mathrm{Mi}$ nuten liegen. Neuere Operationstechniken (Netze) führen zu deutlich geringeren postoperativen Schmerzen, als dies früher der Fall war. Auch hier werden sich die meisten ambulant tätigen Anästhesisten wohl zu einer ambulanten Operation bekennen. Gegebenenfalls sollte postoperativ der Blutzucker kontrolliert werden, eine Oberkörperhochlagerung postoperativ erleichtert die Atmung. Auf die Gabe atemdepressiv wirkender Schmerzmedikamente kann in der Regel verzichtet werden. 


\section{FALLBEISPIEL}

\section{Ambulante OP fraglich}

Wie sieht die Sachlage aus, wenn bei unserer Patientin eine kosmetische Bauchdeckenstraffung durchgeführt werden soll? In diesem Fall legen die Komorbiditäten einen stationären Eingriff nahe, wenn man

- eine Operationsdauer von 2-3 Stunden unterstellt (die je nach Befund und Operateur auch deutlich länger sein kann),

- eine gering erhöhte Blutungsneigung aufgrund der Einnahme von ASS (Z. n. Koronarstent) annimmt und

- die chronische Bronchitis mit ins Kalkül zieht.

In der Praxis werden sich auch hier viele Anästhesisten für die Möglichkeit der ambulanten Durchführung aussprechen, wobei die vorhandene Infrastruktur ein entscheidendes Kriterium darstellt. Die Fragen, die es zu beantworten gilt, lauten:

- Kann man sich auf die Einschätzungen des Operateurs verlassen?

- Macht der Patient - unabhängig von den Untersuchungsbefunden - einen stabilen Eindruck?

- Ist der Patient für diesen ambulanten Eingriff motivierbar (wurde in unserem Fall vorausgesetzt)?

- Kann der Patient postoperativ länger überwacht werden?

- Kann man den Patienten ggf. in der Einrichtung übernachten lassen?

- Ist ein Krankenhaus in erreichbarer Nähe?

- Ist ggf. postoperativ eine CPAP-Beatmungsmaske vorhanden?

\section{Merke}

Ein abendlicher Anruf des Operateurs oder Anästhesisten beim Patienten sollte Routine sein, ebenso die Mitgabe einer Notfalltelefonnummer, unter der einer der beteiligten Ärzte nachts erreichbar ist.

An den Beispielen („Ambulante OP möglich“ und „Ambulante OP fraglich“) sollte deutlich werden, dass die Entscheidung ambulant oder stationär nicht von 1 oder 2 Faktoren abhängt. Wie immer bestimmt die Erfahrung von Operateur und Anästhesist die Grenzen der ambulanten Möglichkeiten.

\section{Ökonomische Grenzen und Möglichkeiten}

\section{Betriebswirtschaftliche Überlegungen}

Die Honorare für niedergelassene Ärzte werden über die Kassenärztlichen Vereinigungen grundsätzlich nach Maßgabe des EBM ausgeschüttet. Viele der Leistungen, die am Patienten vollbracht werden, sind dabei budgetiert, teils auch unterfinanziert. Das ambulante Operieren ist dabei einer der Bereiche, die nicht budgetiert sind und über die Kassenärztlichen Vereinigungen den Kostenträgern direkt in Rechnung gestellt werden. Aufgrund dieser Struktur ist das ambulante Operieren eines der wenigen Gebiete, auf denen der Vertragsarzt zusätzliches Honorar generieren kann. Selbstverständlich gilt das zuvor Gesagte auch für Krankenhäuser, wobei bei diesen aus Sicht der Autoren weniger das pekuniäre Interesse im Vordergrund steht. Hier geht es eher darum, dass über ambulante Operationen der Weg des Patienten hin zur eigenen Einrichtung gebahnt werden soll. Beide Beweggründe beinhalten einen „Moral Hazard“, d. h. Fehlanreize für die Indikationsstellung.

Beide - sowohl Krankenhaus als auch niedergelassener Vertragsarzt - müssen langfristig Gewinne machen, sonst werden sie vom Markt verschwinden. An dieser Stelle sei jedoch darauf hingewiesen, dass der Gewinnbegriff des Krankenhauses sich deutlich von dem des niedergelassenen Arztes unterscheidet: Beim Krankenhaus werden die Gewinne nach Abzug der Lohnkosten ausgewiesen - beim niedergelassenen Vertragsarzt müssten aus seinem Gewinn noch der Unternehmerlohn und Sozialabgaben in Abzug gebracht werden, um eine Vergleichbarkeit herzustellen.

Einerseits werden die Bestimmungen hinsichtlich Hygiene, Zertifizierung, Arbeitsschutz, Patientenschutz usw. immer restriktiver, anderseits ist die jährliche Anpassung der Vergütung nach EBM oder GOÄ nur gering. Dadurch wird die Gewinnmarge in allen Bereichen zunehmend geringer. Eine Steigerung des Gewinns ist somit nur über eine Steigerung der Fallzahlen beim ambulanten Operieren darstellbar oder durch das - nicht gewollte und ggf. ungesetzliche - Absenken von Standards. Beide Vorgehensweisen bergen Risiken in sich und sind nicht gewollt. Ein Absenken von Standards darf auf keinen Fall erfolgen, um das Patientenrisiko nicht zu erhöhen. Eine Zunahme ambulanter Operationsfälle ist erwünscht, jedoch eher durch Verlagerung von stationären Eingriffen in den ambulanten Bereich als durch eine Erhöhung der Gesamtfallzahl. 


\section{Volkswirtschaftliche Überlegungen}

Die 2010 in Bayern durchgeführte Oberender-Studie hat gezeigt: Im ambulanten Operieren steckt großes volkswirtschaftliches Potenzial. Aus verschiedenen Fachbereichen und nur für einige wenige Operationen wurde der Erlös aus Eingriffen verglichen - einmal berechnet als stationärer Eingriff nach dem DRG-System, zum anderen berechnet nach EBM und $\S 115 \mathrm{~b}$ SGB V. Je nach Szenario wurden Einsparpotenziale von 25 bis 515 Mio. Euro errechnet [25].

Amerikanische Untersuchungen zeigen, dass durch Multiplikatoreffekte auch die Region, in der sich die ambulante Operationseinrichtung befindet, profitiert. Die Nachfrage durch die ambulante Operationseinheit führt zu höheren Gewinnen lokaler Zulieferer und somit mittelbar zu höheren Steuereinnahmen [26].

\footnotetext{
Merke

Nosokomiale Infektionen lassen sich vermutlich niemals ganz vermeiden. Auf dem Feld der ambulanten Chirurgie sind sie jedoch extrem selten.
}

In Deutschland erkranken jährlich zwischen 400000 und 600000 Menschen an einer nosokomialen Infektion und 10000 bis 15000 Patienten versterben daran [27]. Eine Hochrechnung der Deutschen Gesellschaft für Krankenhaushygiene e.V. geht sogar von 800000 bis 1,2 Mio. nosokomialen Infektionen und 20000 bis 30000 Todesfällen pro Jahr in Deutschland aus [28]. Das bedeutet insgesamt einen großen volkswirtschaftlichen Schaden durch Krankheitstage, Krankentagegeld und letztlich Berufsunfähigkeit. In diesem Zusammenhang verwundert es, dass das ambulante Operieren nicht flächendeckend besser finanziert ist. Denn nicht das Operieren im Grenzkostenbereich (also zu einer Vergütung, die nur noch die variablen Kosten deckt) sollte das volkswirtschaftliche Ziel sein - sondern vielmehr ein Arbeiten mit Gewinnen, die Neuinvestitionen ermöglichen.

\section{Fazit}

Betriebs- und volkswirtschaftlich bieten ambulante Operationen eher Möglichkeiten als Grenzen: Für den niedergelassenen Vertragsarzt stellen sie eine der wenigen Möglichkeiten zur Umsatzsteigerung dar, für das Krankenhaus beinhalten sie neben dem monetären Aspekt auch einen Werbeaspekt. Nach Ansicht des BDA, des BAO und der Deutschen Krankenhausgesellschaft ist das System des ambulanten Operierens deutlich unterfinanziert, sodass noch längst nicht alle Möglichkeiten ausgeschöpft sind.

\section{KERNAUSSAGEN}

- Die Anzahl ambulanter Operationen in Deutschland ist steigend. Dieser Trend lässt sich seit Jahren beobachten. Voraussetzung ist eine leitliniengerechte Infrastruktur.

- Die persönliche Kompetenz des Anästhesisten darf sich nicht nur auf den Facharztstandard beschränken, sondern der Arzt sollte Erfahrung in der ambulanten Anästhesie haben.

- Gut eingestellte Komorbiditäten des Patienten stellen in der Regel keine Kontraindikation für eine ambulante Durchführung dar.

- Auch der schwer übergewichtige Patient kann ambulant operiert werden, sofern er compliant ist, Komorbiditäten gut eingestellt sind und die intensive postoperative Betreuung gewährleistet ist.

- Ein obstruktives Schlafapnoe-Syndrom ist per se keine Kontraindikation für die ambulante Durchführung des Eingriffs. Wichtig sind auch hier die intensive postoperative Betreuung und das Vorhandensein eines CPAP-Gerätes schon im Aufwachraum.

- Ein wichtiges Entscheidungskriterium bei kardial vorbelasteten Patienten ist die Bestimmung des metabolischen Äquivalents (MET). Ein MET-Wert größer 4 wird als ausreichend für ambulante Eingriffe angesehen.

- Postoperativ kann der Patient in Begleitung nach Hause entlassen werden, wenn Chirurg und Anästhesist sich vom Zustand des Patienten überzeugt haben.

- Eine verständige Begleitperson und der Hinweis an den Patienten, dass er nicht aktiv am Straßenverkehr teilnehmen darf, sind für die Entlassung unabdingbar.

- Die Entscheidung ambulant oder stationär muss immer patientenindividuell getroffen werden. Eine generelle Aussage, welcher Eingriff wie durchgeführt wird, kann nicht getroffen werden.

\section{Interessenkonflikt}

Die Autoren erklären, dass kein Interessenkonflikt besteht. 


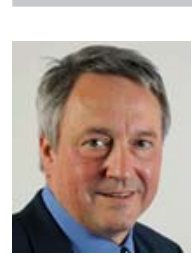

\section{Harald Hofer}

Dr. med. Jahrgang 1957. 1977-1984 Studium der Humanmedizin an der Friedrich-AlexanderUniversität Erlangen-Nürnberg. 1985-1989 Facharztausbildung Anästhesie. Seit 1990 niedergelassen als Facharzt für Anästhesiologie in Dinslaken. Landesvorsitzender des BDA in Nordrhein.

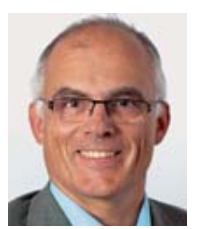

\section{Frank Vescia}

Dr. med. Jahrgang 1961. 1981-1987 Studium der Humanmedizin an der Johannes-Gutenberg-Universität Mainz. 1987-1989 Chirurgische Weiterbildung KH Pfullendorf, 1989-1994 Facharztausbildung Anästhesie in Singen/Hohentwiel und Universitätsklinikum Regensburg. Seit 1995 niedergelassen als Facharzt für Anästhesiologie, stellvertretender Landesvorsitzender des BDA in Bayern und Vizepräsident des BDA.

\section{Korrespondenzadresse}

Dipl.-Volkswirt Dr. med. Harald Hofer

Arzt für Anästhesiologie

Scheepermannsweg 32

46535 Dinslaken

Dr.Hofer@narkose.de

\section{Wissenschaftlich verantwortlich} gemäß Zertifizierungsbestimmungen

Wissenschaftlich verantwortlich gemäß Zertifizierungsbestimmungen für diesen Beitrag ist Dr. med. Harald Hofer, Dinslaken.

\section{Literatur}

[1] Waters RM. The down-town anesthesia clinic. Am J Surg (Anesth Suppl) 1919; 33: 71-73

[2] Kassenärztliche Bundesvereinigung (KBV). Einheitlicher Bewertungsmaßstab EBM. 2017. Im Internet: http://www.kbv. de/html/online-ebm.php; Stand: 21.08.2017

[3] Bundesministerium der Justiz und für Verbraucherschutz. Sozialgesetzbuch (SGB) Fünftes Buch (V) - Gesetzliche Krankenversicherung - §115b Ambulantes Operieren im Krankenhaus. Im Internet: http://www.gesetze-im-internet.de/sgb_5/ _ 115b.html; Stand: 21.08.2017

[4] Bundesverband für Ambulantes Operieren e.V. Ambulantes Operieren - Eingriffe. Im Internet: http://www.operieren.de/ content/e3224/e10/e589/; Stand: 21.08.2017

[5] Standl T, Lussi C. Ambulantes Operieren in Klinik, Praxis und MVZ. 3. Aufl. Berlin: Springer; 2016

[6] Deutsche Gesellschaft für Anästhesiologie und Intensivmedizin e.V. und Berufsverband Deutscher Anästhesisten e.V. Mindestanforderungen an den anästhesiologischen Arbeitsplatz. Anästh Intensivmed 2013; 54: 39-42

[7] Deutsche Gesellschaft für Anästhesiologie und Intensivmedizin (DGAI). Entschließungen, Empfehlungen, Vereinbarungen. Im Internet: http://www.dgai.de/publikationen/vereinbarungen; Stand: 21.08.2017
[8] Shore JW, Menke AM, Kelley B. New safety rules for outpatient surgery. OMIC Digest Fall 2005; 15: 4

[9] Deutsche Gesellschaft für Anästhesiologie und Intensivmedizin, Deutsche Gesellschaft für Chirurgie, Deutsche Gesellschaft für Innere Medizin. Präoperative Evaluation erwachsener Patienten vor elektiven, nicht kardiochirurgischen Eingriffen. 2011. Im Internet: http://www.bda.de/docman/alle-dokumente-fuer-suchindex/oeffentlich/empfehlungen/526praeoperative-evaluation-erwachsener-patienten-vor-elektiven-nicht-kardiochirurgischen-eingriffen.html; Stand: 21.08.2017

[10] SpringerMedizin.at. Päoperative Optimierung. Der pulmonal beeinträchtigte Patient mit COPD. 2010. Im Internet: http:// www.springermedizin.at/artikel/20049-praeoperative-optimierung; Stand: 21.08.2017

[11] Mahmoud KD, Sanon S, Habermann EB et al. Perioperative cardiovascular risk of prior coronary stent implantation among patients undergoing noncardiac surgery. J Am Coll Cardiol 2016; 67: 1038-1049

[12] Deutsche Gesellschaft für Kardiologie, European Society of Cardiology. Präoperative kardiale Risikoeinschätzung und perioperatives kardiales Management bei nichtkardialen chirurgischen Eingriffen. 2011. Im Internet: http://leitlinien.dgk.org/ files/2011_Pocket-Leitlinien_Risikoeinschaetzung_Management.pdf; Stand: 21.08.2017

[13] Deutsche Adipositas Gesellschaft. Gewichtsklassifikation bei Erwachsenen anhand des BMI (nach WHO, 2000). 2012. Im Internet: http://www.adipositas-gesellschaft.de/index.php? id=39; Stand: 21.08 .2017

[14] Joshi GP, Ahmad S, Riad W et al. Selection of obese patients undergoing ambulatory surgery: a systematic review of the literature. Anesth Analg 2013; 117: 1082-1091

[15] Hoell J, Sheboygan A. Memorial Medical Center. Ambulatory Surgery Center - Patient Criteria and Selcetion. Im Internet: http://www.thewsa.org/files/9314/7342/9062/ WSA_16AM_Handout_Hoell.pdf; Stand: 21.08.2017

[16] Dobrowolska-Zarzycka M, Dunin-Wilczynska I, Mitura I et al. $\mathrm{BMI}$ in patients with obstructive sleep apnea. Curr Issues Pharm Med Sci 2015; 28: 244-246

[17] Pulz S. Nachrichten aus der internationalen Fachliteratur: ASALeitlinien: OSAS-Patienten (Anesthesiology 2014; 120: 268286). Anästhesiol Intensivmed Notfallmed Schmerzther 2014; 49: 572

[18] The American Society of Anesthesiologists Committee on Standards and Practice Parameters and the Task Force on Perioperative Management of Obstructive Sleep Apnea. Practice Guidelines for the Perioperative Management of Patients with Obstructive Sleep Apnea. Anaesthesiology 2014; 120: 268286

[19] Dean A. Best Practices for Proper Care of the Morbidly Obese in Surgery Centers. 2012. Im Internet: http://www.beckersasc. com/asc-accreditation-and-patient-safety/best-practices-forproper-care-of-the-morbidly-obese-in-surgery-centers.html; Stand: 21.08.2017

[20] Kretz F], Teufel F, Hrsg. Anästhesie und Intensivmedizin. Berlin: Springer; 2006: 314

[21] Heck M, Fresenius M, Busch C. Klinikmanual Anästhesie. 2. Aufl. Berlin: Springer; 2015: $521 \mathrm{f}$

[22] The Pennsylvania Bulletin. Rules and Regulations. Title 28 Health and Safety. Department of Health [28 PA. Code CHS. $551,553,555,557,559,561,563,565,567,569,571$ and 573]. Ambulatory Surgical Facilities [29 Pa.B. 5583]. Im Internet: http://www.pabulletin.com/secure/data/vol29/29-43/ 1827.html; Stand: 21.08.2017 
[23] Vereinbarung des Berufsverbandes Deutscher Anästhesisten und des Berufsverbandes der Deutschen Chirurgen. Zusammenarbeit bei der operativen Patientenversorgung. Anästh Intensivmed 2016; 57: 213-215

[24] BGH. Urteil vom 08.04.2003, AZ: VI ZR 265/02. Im Internet: http://www.openjur.de/u/66710.html; Stand: 21.08.2017

[25] Bundesverband für ambulantes Operieren e.V. Gutachten Oberender \& Partner. Ökonomische Betrachtung des Ambulanten Operierens. 2010. Im Internet: http://www.kvb.de/fileadmin/kvb/dokumente/Praxis/Alternative-Versorgungsformen/Weitere/KVB-Amb-OP-Studie-Oberlaender100401.pdf; Stand: 21.08.2017

[26] Schneider JE, Dumville A. The Economic Impact of Ambulatory Surgery Centers in Washington. 2010. Im Internet: http://wasca.net/wp-content/uploads/2010/02/WASH-Economic-Impact-Study-FINAL-Email-2.pdf; Stand: 21.08.2017
[27] Gastmeier P, Geffers C. Nosokomiale Infektionen in Deutschland: Wie viele gibt es wirklich? Eine Schätzung für das Jahr 2006. Dtsch Med Wochenschr 2008; 133: 1111-1115

[28] Walger P, Popp W, Exner M. Stellungnahme der DGKH zu Prävalenz, Letalität und Präventionspotenzial nosokomialer Infektionen in Deutschland 2013. Hyg Med 2013; 38: 329-338

\section{Bibliografie}

DOI https://doi.org/10.1055/s-0042-120247

Anästhesiol Intensivmed Notfallmed Schmerzther 2017; 52: 666-678 @ Georg Thieme Verlag KG Stuttgart · New York ISSN 0939-2661 


\section{Punkte sammeln auf CME.thieme.de}

Diese Fortbildungseinheit ist 12 Monate online für die Teilnahme verfügbar.

Sollten Sie Fragen zur Online-Teilnahme haben, finden Sie unter cme.thieme.de/hilfe

eine ausführliche Anleitung. Wir wünschen viel Erfolg beim Beantworten

der Fragen!

Unter eref/thieme.de/ZZWHM6S oder über den QR-Code kommen Sie direkt zum Artikel zur Eingabe der Antworten.

VNR 2760512017152374064

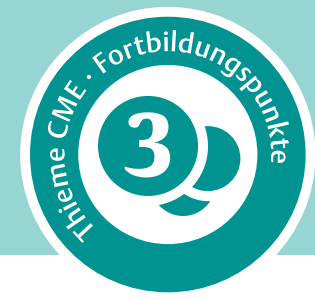

\section{Frage 1}

Welche Aussage zu ambulanten Anästhesien ist richtig?

A Patienten mit Hypertonie können nicht ambulant operiert werden.

B Nach Implantation von Koronarstents dürfen elektive Eingriffe immer erst nach 1 Jahr durchgeführt werden.

C Ein BMI von 42 stellt eine absolute Kontraindikation für einen ambulanten Eingriff dar.

D Ein ambulanter Eingriff darf nur durchgeführt werden, wenn bestimmte apparative Standards eingehalten werden.

E Auf einen Defibrillator kann im ambulanten Setting verzichtet werden.

\section{Frage 2}

Welche Aussage zur apparativen Ausstattung des anästhesiologischen Arbeitsplatzes ist richtig?

A Die Kriterien legt der einzelne Anästhesist fest.

B Die von DGAI und BDA gelisteten Mindestanforderungen müssen eingehalten werden.

C Ein Relaxometer ist auf jeden Fall vorzuhalten.

D Ohne oszillometrische Blutdruckmessung ist eine Narkose nicht möglich.

E Auf einen EKG-Monitor kann verzichtet werden.

\section{Frage 3}

Welche Aussage zur Adipositas permagna ist richtig?

A Die Lungenfunktion ist unverändert.

B Jeder Operationstisch ist für hohe Gewichte zugelassen.

C Es besteht kein erhöhtes Thromboserisiko.

D Eine diabetische Stoffwechsellage ist unabhängig von der Adipositas permagna.

E Sofern Anästhesiemedikamente nach dem Körpergewicht des Patienten berechnet werden, erfolgt beim adipösen Patienten eher eine Über- als eine Unterdosierung.

\section{Frage 4}

Welche der Aussagen zur betriebswirtschaftlichen Betrachtung ambulanter Operationen ist richtig?

A Die Vergütung ambulanter Operationen in Deutschland ist ausreichend.

B GOÄ und EBM bilden alle ambulanten Operationen mit Preisen korrekt ab.

C Ein Operieren zu Grenzkosten kann in die Insolvenz führen.

D Ein „Moral Hazard“ beim ambulanten Operieren existiert nicht.

E Die einzelnen Leistungen werden im DRG-System mit denselben Honoraren wie im EBM vergütet.

\section{Frage 5}

Welche Aussage zur postoperativen Überwachung ist richtig?

A Die unmittelbare postoperative Überwachung kann nach ambulanten Operationen in die Hände von unerfahrenem Pflegepersonal gelegt werden

B Ein Hinweis auf die eingeschränkte Verkehrstüchtigkeit kann unterbleiben, da dies der Patient auf dem Einwilligungsbogen unterschrieben hat.

C Der Patient kann auch ohne Begleitung nach Hause gehen.

D Eine verständige Begleitperson sollte zumindest bis zum nächsten Morgen anwesend sein.

E Die Entlassung des Patienten ist ausschließlich Sache des Anästhesisten.

\section{Frage 6}

Welche Aussage zum OSAS (obstruktives Schlafapnoe-Syndrom) trifft zu?

A Patienten mit OSAS können nicht ambulant operiert werden.

B Adipositas und OSAS sind 2 immer getrennt auftretende $\mathrm{Er}$ krankungen.

C OSAS-Patienten mit Adipositas können bei gut eingestellten Begleiterkrankungen ambulant operiert werden.

D Zentral wirkende Analgetika können uneingeschränkt postoperativ verwendet werden.

E Das Vorhalten einer CPAP-Maske ist im ambulanten Bereich entbehrlich.

- Weitere Fragen auf der folgenden Seite... 


\section{Punkte sammeln auf CME.thieme.de}

Fortsetzung $\ldots$

\section{Frage 7}

Welche Aussage zur maximalen Operationsdauer für ambulante Eingriffe ist richtig?

A In Deutschland ist die Maximaldauer auf 2 Stunden begrenzt.

$B$ In den USA sind die Maximalzeiten in allen Staaten auf 4 Stunden begrenzt.

C Eine Operationsdauer über 1 Stunde führt vermehrt zu Komplikationen.

D In Deutschland gibt es keine gesetzlichen Vorgaben für die Länge einer geplanten ambulanten Narkose/Operation.

E Die maximale Operationsdauer in Deutschland legen DGAI und BDA fest.

\section{Frage 8}

Welche Aussage zu einer ambulanten Operation über 6 Stunden ist richtig?

A Es muss auf die Blasenentleerung geachtet werden.

B Ambulante Operationen über 6 Stunden sind in Deutschland verboten.

C Das Wärmemanagement ist von untergeordneter Bedeutung.

D Lagerungsschäden treten bei ambulanten Operationen nicht auf.

E Die Infrastruktur des Operationszentrums ist unerheblich.

\section{Frage 9}

Welche Aussage zum BMI ist richtig?

A Ein Patient mit BMI $=34$ ist im Stadium Adipositas Grad II.

$B$ Ein Patient mit einem $\mathrm{BMI}<25$ ist untergewichtig.

C Ein Patient mit einem $\mathrm{BMI}=55$ ist im Stadium Adipositas Grad III.

D Bei einem Patienten mit BMI $=40$ treten immer Intubationsschwierigkeiten auf.

E Ein Patient mit BMI $=40$ hat im Regelfall keine eingeschränkte Lungenfunktion.

\section{Frage 10}

Welche Aussage zu volkswirtschaftlichen Überlegungen hinsichtlich ambulanter Eingriffe ist richtig?

A Ambulante Eingriffe haben dieselbe Rate an nosokomialen Infektionen wie stationäre Eingriffe.

B Eine Ausweitung ambulanter Eingriffe könnte zu Einsparungen der Krankenkassen in Millionenhöhe führen.

C Der volkswirtschaftliche Schaden durch im Krankenhaus erworbene Infektionen ist gering.

D Der volkswirtschaftliche Vorteil ambulanter Eingriffe verglichen mit stationären Eingriffen ist nicht bewiesen.

E Nosokomiale Infektionen gibt es überwiegend im ambulanten Bereich. 